\title{
Probable genetic Creutzfeldt-Jakob Disease with rare E196K mutation
}

\author{
Katarzyna Orlewska ${ }^{1}$, Monika Błońska ${ }^{1}$, Łukasz Błoński ${ }^{1}$, Łukasz Madej $^{2}$, Justyna Klusek ${ }^{2}$ \\ ${ }^{1}$ Department of Internal Medicine, Tytus Chałubiński’s Hospital, Zakopane, Poland \\ ${ }^{2}$ Department of Surgical Medicine, with Laboratory of Medical Genetics, Collegium Medicum, \\ Jan Kochanowski University, Kielce, Poland
}

Key words: genetic Creutzfeldt-Jakob Disease, E196K mutation

(Neurol Neurochir Pol 2021; 55 (1): 113-114)

\section{To the Editors:}

Inherited prion diseases (IPD) are characterised by the accumulation of pathological prion protein in the central nervous system resulting from a mutation of the prion protein (PRNP) gene. More than 50 PRNP mutations have been described, and they account for approximately $10 \%$ of all cases of prion diseases in humans [1]. Three forms of IPD are specified based on clinical and genetic features: genetic Creutzfeldt-Jakob Disease (gCJD), fatal familial insomnia, and Gerstmann-Sträussler-Scheinker Disease [2].

We report the first Polish case of a patient with a rare E196K mutation who presented with clinical features of CJD.

A 66-year-old woman presented to hospital with a 10-day history of memory loss, confusion, behavioural disorders and hypertension. Her medical history was otherwise irrelevant. According to her daughter, the patient had undergone an epileptic seizure with locked jaw on the previous day. In retrospect, family members admitted to having observed visual hallucinations and stereotypy (manifesting in hair brushing) in the preceding days. Upon admission, the patient was supine, conscious, allo- and autopsychically disoriented, cardiovascularly and respiratorily stable, with limited verbal contact, but able to follow simple commands. Based on initial EEG results, she has been admitted to the neurology ward with a suspicion of nonconvulsive status epilepticus. Neurological examination revealed motor and sensory aphasia, orofacial dyskinesia, myoclonus in the right lower limb, and positive right Babinski sign. During hospitalisation, the patient developed spastic quadriplegic hypertonia and intermittent myoclonic jerking.
Repeated EEG revealed theta waves in the frontal, temporal and occipital regions bilaterally, with a left side dominance; paroxysmal, occasionally periodic slow waves and periodic sharp-wave complexes (Supplementary Fig. 1). Noncontrast head computed tomography scan was negative for any acute intracranial pathology. Doppler ultrasound of the internal carotid and vertebral arteries revealed multiple, haemodynamically irrelevant atherosclerotic plaques. Magnetic resonance imaging (MRI) showed several non-specific white matter lesions and mild periventricular leukoaraiosis, as well as diffusion restriction involving the frontal and temporal cortex in the left hemisphere and basal ganglia bilaterally (Fig. 1). Routine laboratory parameters were unremarkable, and cerebrospinal fluid (CSF) analysis revealed no white blood cells, normal glucose levels, and mildly elevated protein $(66 \mathrm{mg} / \mathrm{dl})$, which proved inconclusive for any acute infectious or metabolic etiology. Other differential diagnoses, including liver and kidney dysfunction and polypharmacy, were excluded.

Given the clinical picture and frequency of occurrence, Alzheimer's Disease and Lewy body dementia were considered. However, MRI findings characteristic for CJD led to a further investigation of the CSF, which confirmed the presence of the 14-3-3 protein. Blood samples were subsequently collected for genetic analysis. The coding region of PRNP was amplified by polymerase chain reaction and then analysed by direct sequencing. This revealed the E196K mutation leading to a substitution of glutamic acid to lysine at codon 196 and homozygosity for methionine at codon 129. The patient died three months after the onset of disease, and no brain autopsy was performed. Probable CJD was diagnosed on the basis of 


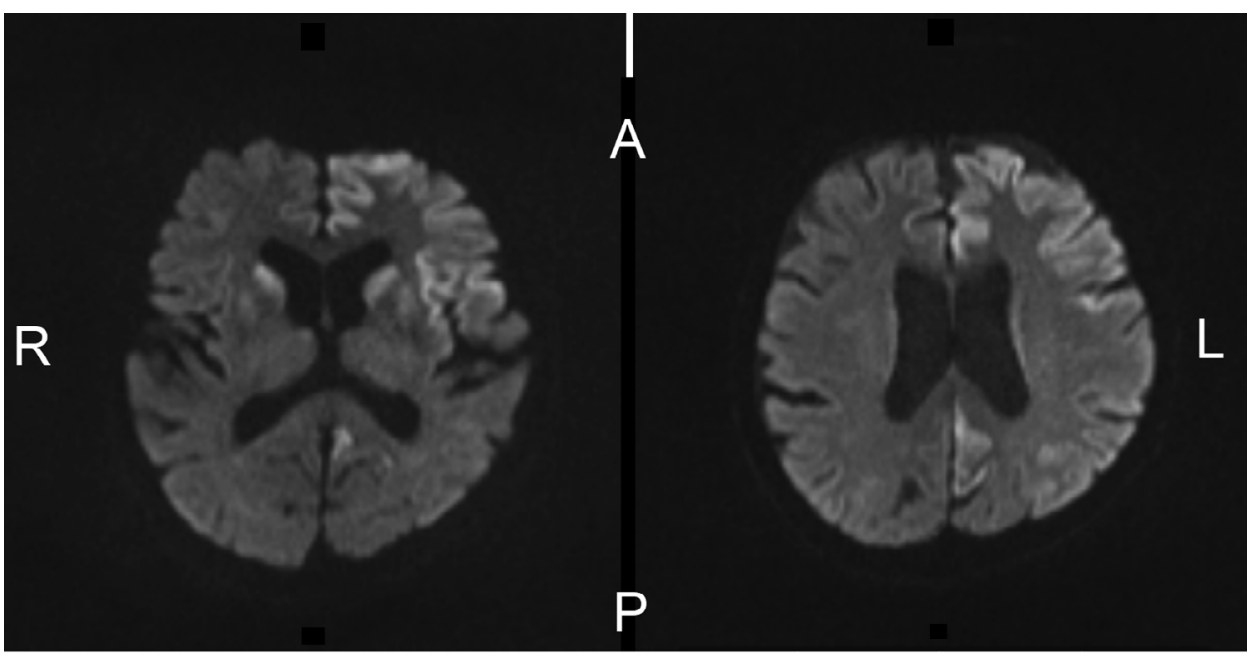

Figure 1. Axial diffusion-weighted MRI at admission

clinical findings and genetic analysis. Due to lack of brain material, a definite diagnosis of CJD was unfeasible.

Because the number of reported E196K mutation cases is low, interpretations of the findings should be made with caution. Nonetheless, the E196K mutation has been neuropathologically confirmed to result in IPD [3], and diffusion-weighted MRI abnormalities have previously been observed in gCJD [4].

The presented case supports the presence of abnormal behaviour among patients with the E196K mutation [5], as well as initial extrapyramidal symptoms and myoclonus with rapid disease progression to akinetic mutism $[6,7]$. Furthermore, a tendency to short disease duration (median 5 months), more frequent occurrence of codon 129 methionine homozygosity [6], and CSF positive for 14-3-3 protein [8] have been observed among E196K mutation carriers, which is in line with our findings.

In conclusion, we report the first Polish case of probable gCJD with a rare E196K mutation. The patient's mother died in similar circumstances, though no clear family history was available. Mutations in the PRNP vary in penetrance; therefore, the majority of patients with gCJD have no family history of neurodegeneration or uncharacterised neurological abnormalities $[6,7]$.

Our study further suggests that PRNP gene sequencing could play a substantial role in identifying hereditary forms of prion diseases [9]. We hope that the presented case will result in greater awareness of the E196K mutation in the PRNP gene as a cause of probable gCJD.

Ethical permission: Ethical approval was not necessary for the preparation of this article.

Acknowledgements: We thank the patient and her family for supplying the required information and donating specimens. Conflicts of interest: None.

Funding: Project financed under the Ministry of Science and Higher Education 'Regional Initiative of Excellence' programme covering the years 2019-2022, project no 024/ RID/2018/19.

\section{References}

1. Jeong BH, Kim YS. Genetic studies in human prion diseases. J Korean Med Sci. 2014; 29(5): 623-632, doi: 10.3346/jkms.2014.29.5.623, indexed in Pubmed: 24851016.

2. Di Fede G, Catania M, Atzori C, et al. Clinical and neuropathological phenotype associated with the novel V189I mutation in the prion protein gene. Acta Neuropathol Commun. 2019; 7(1): 1, doi: 10.1186/ s40478-018-0656-4, indexed in Pubmed: 30606247.

3. Eigenbrod S, Frick P, Giese A, et al. Comprehensive neuropathologic analysis of genetic prion disease associated with the E196K mutation in PRNP reveals phenotypic heterogeneity. J Neuropathol Exp Neurol. 2011; 70(3): 192-200, doi: 10.1097/NEN.0b013e31820cd8a4, indexed in Pubmed: 21293298.

4. Nitrini R, Mendonça RA, Huang $\mathrm{N}$, et al. Diffusion-weighted MRI in two cases of familial Creutzfeldt--Jakob disease. J Neurol Sci. 2001; 184(2): 163-167, doi: 10.1016/s0022-510x(01)00432-4, indexed in Pubmed: 11239951.

5. Béjot Y, Osseby GV, Caillier M, et al. Rare E196K mutation in the PRNP gene of a patient exhibiting behavioral abnormalities. Clin Neurol Neurosurg. 2010; 112(3): 244-247, doi: 10.1016/j.clineuro.2009.11.002, indexed in Pubmed: 20005032.

6. Krasnianski A, Heinemann U, Ponto $\mathrm{C}$, et al. Clinical findings and diagnosis in genetic prion diseases in Germany. Eur J Epidemiol. 2016; 31(2): 187-196, doi: 10.1007/s10654-015-0049-y, indexed in Pubmed: 26076917.

7. Shi Qi, Chen C, Song XN, et al. A Chinese Creutzfeldt-Jakob disease patient with E196K mutation inPRNP. Prion. 2014; 5(2): 117-120, doi: 10.4161/pri.5.2.15846.

8. Shi Qi, Zhou W, Chen C, et al. Rare E196A mutation in PRNP gene of 3 Chinese patients with Creutzfeldt-Jacob disease. Prion. 2016; 10(4): 331-337, doi: 10.1080/19336896.2016.1190897, indexed in Pubmed: 27310471.

9. Zimowski J, Kulczycki J, Lojkowska W, et al. Hereditary form of prion disease in Poland. Neurol Neurochir Pol. 2012; 46(6): 509-518, doi: 10.5114/ninp.2012.32353, indexed in Pubmed: 23319218. 